\title{
Gravitational waves as tracers of nuclear equation of state
}

\section{Ruben M. Cabezon ${ }^{1}$}

Universitat Politecnica de Catalunya

Jordi Girona, 1, 08034 Barcelona, Spain

E-mail: ruben. cabezon aupc. edu

\section{Domingo Garcia-Senz}

Universitat Politecnica de Catalunya

Jordi Girona, 1, 08034 Barcelona, Spain

E-mail: domingo.garcialupc.edu

The signal of neutron star (NS) mergers has the imprint of the EOS of dense nuclear matter, which is still not well known. A set of gravitational waves (GW) signals have been calculated from 3D hydrodynamical simulations of NS-NS mergers using the Smoothed Particle Hydrodynamics technique (SPH) with different EOS. By analyzing the morphology and time evolution of the signal we want to be able to discriminate among the variety of proposed EOS after a successful GW detection has been done.

Supernovae: lights in the darkness (XXIII Trobades Cientifiques de la Mediterrània) Mao, Menorca, Spain

3-5 October, 2007

\footnotetext{
1 Speaker
} 


\section{Introduction}

Compact objects are systems with an unquestionable scientific interest due to their extreme physical properties: high densities, strong gravitational fields, quantum degeneration of the material. Among the scenarios that involve compact objects we are interested in double neutron star (DNS) mergers, which are powerful emitters of gravitational waves. This emission can be used to put strong constraints in one important unknown property of neutron stars: their equation of state (EOS hereafter). This is interesting not only to improve the knowledge about the astrophysical processes where neutron stars are involved in, but also to understand how matter behaves under extreme conditions of high pressure and density.

It has been shown [1] that there are specific features in the gravitational wave emission that are connected to different hydrodynamical stages of the coalescence. The outcome depends on the compactness of the neutron stars involved during the merging and, therefore on their EOS. The relationship can be envisaged in a qualitative way as follows: gravitational emission is produced by the quadrupolar movement of mass distributions, but these mass distributions (and their hydrodynamical evolution) depend very sensitively on the equation of state of the stellar matter. Thus the fingerprint of the EOS is somehow encoded in the gravitational wave emission. How to decode that information has been the subject of intense research in the two last decades.

The other side of the coin is, of course, the successful detection of gravitational waves. The expected amplitude of the displacement due to a gravitational wave produced by a DNS merger scenario is typically of the order of $10^{-21} \mathrm{~m}$. This is an incredible low deformation which requires the development of high technology infrastructures devoted to achieve a successful detection. The most promising detectors for the gravitational wave emission from DNS mergers are ground based interferometers whose frequency band of detection is suitable for these scenarios. The main projects of interferometric detectors are summarized in Table 1. It is worth to say that the most interesting features of the gravitational wave spectra are expected at frequencies beyond $1 \mathrm{kHz}$, that is just beyond the range of sensibility of current detectors. Only with a third generation interferometric detectors like advanced LIGO that range of frequencies will be available to observation. Other possibility could be the use of resonant detectors that have specific high-frequency resonances. The main problem is that these detectors are narrowband instruments so they need to be "tuned" in the frequency of interest, which is not completely known a priori.

The main purpose of this report is to highlight the most distinctive features in the gravitational waves that carry information of the EOS and to identify qualitatively the main differences that appear in the emission depending on the equation of state. To do that we have performed a series of numerical simulations using the Smoothed Particle Hydrodynamics 
technique with eleven different EOS ranging from soft to stiff dependences between pressure and density.

\begin{tabular}{|c|c|c|c|}
\hline NAME (COUNTRY) & LOCATION & ARM & SENS. PEAK \\
\hline LIGO (USA) & Livingston & $4 \mathrm{~km}$ & $3 \cdot 10^{-23}$ at $180 \mathrm{~Hz}$ \\
LIGO (USA) & Hanford & $4 \mathrm{~km}$ & Id \\
TAMA 300 (JAPAN) & Tokyo & $300 \mathrm{~m}$ & $5 \cdot 10^{-21}(0.7-1 \mathrm{kHz})$ \\
GEO 600 (GERMANY) & Hannover & $600 \mathrm{~m}$ & $8 \cdot 10^{-23}$ at $600 \mathrm{~Hz}$ \\
\hline VIRGO (ITALY) & Pisa & $3 \mathrm{~km}$ & $10^{-22}$ at $500 \mathrm{~Hz}$ \\
\hline
\end{tabular}

TABLE 1. Main characteristics of the operating ground-based interferometric detectors of gravitational waves.

\section{DNS coalescence and gravitational waves}

DNS scenarios are usually formed after the evolution of two massive stars in a binary system, with masses greater than $8 \mathrm{M}_{\odot}$. Population synthesis models [2] have identified 14 different evolutionary channels leading to the formation of a DNS system, being the dominant ones those producing tight short lived binaries with one recycled pulsar. Their merger times are typically about $1 \mathrm{Myr}$ and the predicted detection rates for LIGO I and II are $1 \cdot 10^{-2}$ and $6 \cdot 10^{1}$ $\mathrm{yr}^{-1}$ respectively. DNS systems have a high disruption rate during their formation because they have to survive to two supernova explosions and still remain gravitationally bound. At the moment there are 7 known DNS systems, all of them belonging to the dominant evolutionary channel.

Numerical simulations of the coalescence of DNS systems have been performed by many groups and represent a unique tool to explore the hydrodynamical evolution of these scenarios. All of them predict that the frequency spectrum of the gravitational wave emission has great relevance because it can be directly related to hydrodynamical stages.

Initially both NS are orbiting around their center of mass at an orbital distance $\mathrm{D}_{\text {orb }}>>$ $\mathrm{R}_{\mathrm{NS}}$. In this stage each star "sees" the other as a point source of gravitational force; hence the loss of angular momentum carried away by the gravitational waves obliges both NS to follow a spiral trajectory that gets them closer. In this point-mass inspiral trajectory the spectrum of the gravitational wave emission decreases monotonically as $d E / d f \propto f^{-1 / 3}$, a trend which is linked to the coalescence process: as the stars are inspiralling their orbital period decreases, hence they spend less time in a given frequency and therefore the amount of energy for that given frequency decreases too. As the stars get closer the point-mass approximation fails and a tidal torque appears deforming the stars. At this point the NS are rotating faster than what is predicted by the point-mass regime, a behaviour which leads to a gravitational wave spectrum 
that falls below the $-1 / 3$ slope. This is the onset of the hydrodynamical instability that leads to the actual merger of the stars. The frequency at which the spectrum departs from the point-mass inspiral regime is very sensitive to the EOS. Softer EOS produce more compact objects, with higher central densities. Therefore the point-mass approximation is valid at lower orbital distances than for stiffer EOS that produce larger and more homogeneous objects. Thus the frequency at which the spectrum departs from the point-mass inspiral regime is higher for soft EOS than for stiffer ones.

When the stars get in contact they form a temporary barlike structure that has a high quadrupolar moment, producing a broad peak in the gravitational wave spectrum. This peak is higher for softer EOS because the merger is produced at higher orbital velocities and is more violent, whereas for stiffer EOS the merger is smoother.

After this point the merging develops very quickly, within one orbit, and both stars form a massive fast rotating neutron star. The gravitational emission drops rather sharply but centrifugal forces prevent this newly born compact object to be spherically symmetric. Instead it remains triaxial with a mass distribution having a non-zero quadrupolar moment therefore it is still emitting gravitational waves, although they progressively fade as the remnant gains axial symmetry. The system recovers axial symmetry faster if the nuclear EOS is softer due to the higher compressibility of the material. Therefore the gravitational emission after the merger carries also a distinctive signature that is connected to the stiffness of the EOS via its rapidness to decay.

\section{Numerical Scheme}

The Smoothed Particle Hydrodynamics technique (SPH from now on) is widely known in the area of computational astrophysics and it has been successfully applied to a large amount of physical scenarios. SPH is a fully lagrangian hydrocode where a continuous system is discretized in a set of particles (actually, from a mathematical point of view, interpolation points, but with an associated mass) where the physical properties are evaluated as a weighted interpolation within the properties of neighbours particles. The main advantage is that there is no need of a grid to evaluate derivatives, avoiding the problems associated to knots and grid distortions. A review on this technique has been published recently [3].

The particular SPH code that we have used to perform the simulations is a standard one. It has a Leapfrog integrator, commutable to a Runge-Kutta, with adaptive time steps, cubic spline kernel, smoothing length spatial and temporally variable and gravity calculation by multipolar expansion. It is a fully Newtonian code thus the results of our simulations are only indicative. In order to make more precise quantitative predictions a post-newtonian [4] or fully relativistic [5] code should be used. The calculation of the gravitational waves is done following the receipt of [6]. This method is based in the quadrupole approximation which is valid for nearly Newtonian sources (see [7]). The reduced quadrupole is defined by [8] as: 


$$
t_{i j}=\int \rho\left(x_{i} x_{j}-\frac{1}{3} \delta_{i j} x_{k} x^{k}\right) d^{3} x
$$

The metric perturbation is directly related to the second time derivatives of $t_{i j}$ in the transverse-traceless gauge:

$$
h_{i j}^{T T}=\frac{2}{r} \frac{G}{c^{4}} \frac{d^{2} t_{i j}^{T T}}{d t^{2}}
$$

For orthonormal spherical coordinates we can build two basis tensors that lead to the known plus and cross polarization modes of the gravitational waves:

$$
\begin{gathered}
h_{+}=\frac{1}{r} \frac{G}{c^{4}}\left(\ddot{\ddagger}_{\theta \theta}-\ddot{\ddagger}_{\phi \phi}\right) \\
h_{\times}=\frac{2}{r} \frac{G}{c^{4}} \ddot{I}_{\theta \phi}
\end{gathered}
$$

The components of the reduced quadrupole in spherical coordinates are related to the Cartesian ones by [9]:

$$
\begin{gathered}
\ddot{I}_{\theta \theta}=\left(\ddot{I}_{x x} \cos ^{2} \phi+\ddot{t}_{y y} \sin ^{2} \phi+\ddot{I}_{x y} \sin 2 \phi\right) \cos ^{2} \theta+\ddot{I}_{z z} \sin ^{2} \theta \\
-\left(\ddot{I}_{x z} \cos \phi+\ddot{t}_{y z} \sin \phi\right) \sin 2 \theta \\
\ddot{t}_{\phi \phi}=\ddot{I}_{x x} \sin ^{2} \phi+\ddot{t}_{y y} \cos ^{2} \phi-\ddot{I}_{x y} \sin 2 \phi \\
\ddot{t}_{\theta \phi}=-\frac{1}{2}\left(\ddot{I}_{x x}-\ddot{I}_{y y}\right) \cos \theta \sin 2 \phi \\
+\ddot{I}_{x y} \cos \theta \cos 2 \phi+\left(\ddot{t}_{x z} \sin \phi-\ddot{t}_{y z} \cos \phi\right) \sin \theta
\end{gathered}
$$

Once we have the relation between the quadrupolar mass distribution and the gravitational wave emission we can take advantage of the lagrangian nature of our code and implement SPH versions of $\ddot{H}_{i j}$ :

$$
\ddot{t}_{l m}=\frac{2}{3} \sum_{i=1}^{n} m^{i}\left[2 v_{l}^{i} v_{m}^{i}+a_{l}^{i} x_{m}^{i}+x_{l}^{i} a_{m}^{i}+\delta_{l m}\left(v_{l}^{i} v_{m}^{i}+x_{l}^{i} a_{m}^{i}-\left|\vec{v}^{i}\right|^{2}-\vec{x}^{i} \cdot \vec{a}^{i}\right)\right]
$$

Where the subscripts refer to the three Cartesian components, the superscripts label the particle and the summation is over all the particles of the simulation. The $x, v$ and $a$ terms are the components of the position, velocity and acceleration vectors respectively, and $m^{i}$ is the mass of the $i^{\text {th }}$-particle.

Now, using equation (8) we can find the amplitude of both polarizations of the gravitational wave emission directly from magnitudes calculated by the SPH code. 
We have selected eleven realistic $\operatorname{EOS}^{1}$ commonly used in numerical simulations that involve neutron stars (fig. 1). Integrating the structure equations for a fixed mass of $1.4 \mathrm{M}_{\odot}$ we

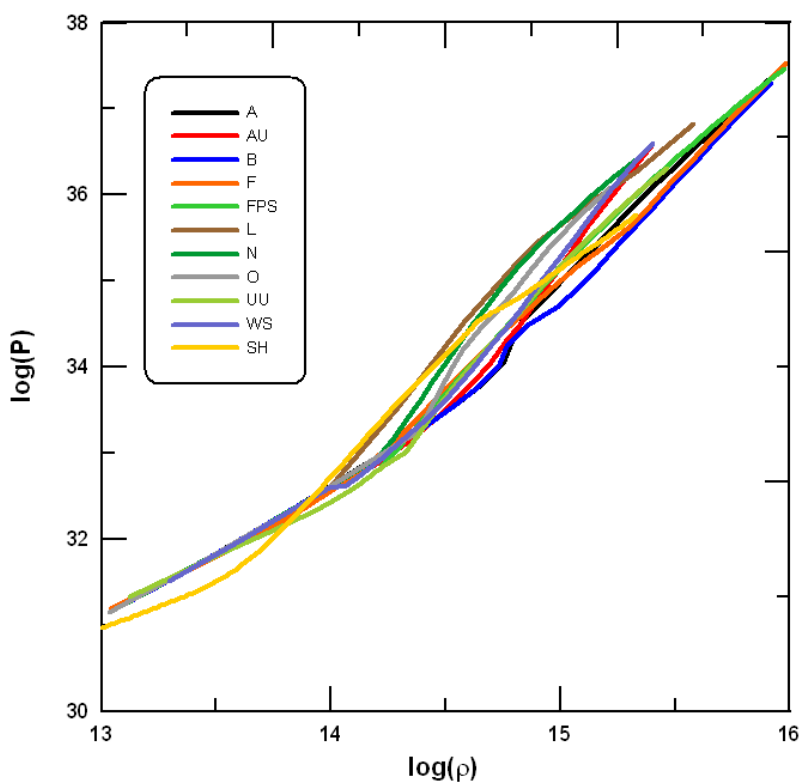

Fig.1. Graphic of the EOS used in this work focusing in the relevant range of densities for neutron stars. obtain the 1D density profile. The 1D profile is mapped to $3 \mathrm{D}$ using 25000 particles for each star. Then each star follows a relaxation process divided in two parts. In the first step particles are allowed to move within a sphere of fixed radius (i.e. the radial component of the velocity is artificially suppressed). By this way the pressure gradients, which have appeared as a numerical artifact due to the random distribution of the particles, are damped out. In a second step particles are allowed to move freely in order to achieve hydrostatic equilibrium.

Following that procedure we obtain the set of stable neutron stars depicted in figure 2 .

From that figure it is clear the influence of the EOS in the structure of a neutron star. Soft EOS lead to more condensed objects because of the high compressibility of the material, while stiff EOS produce a more homogeneous object with lower central densities and greater radius. The central densities ranged from $2.5 \cdot 10^{14}$ to $1.4 \cdot 10^{15} \mathrm{~g} / \mathrm{cm}^{-3}$, while the radius varied from 18 to 11 $\mathrm{km}$.

Once we have the initial model of each star we simulate a double neutron star merger scenario with

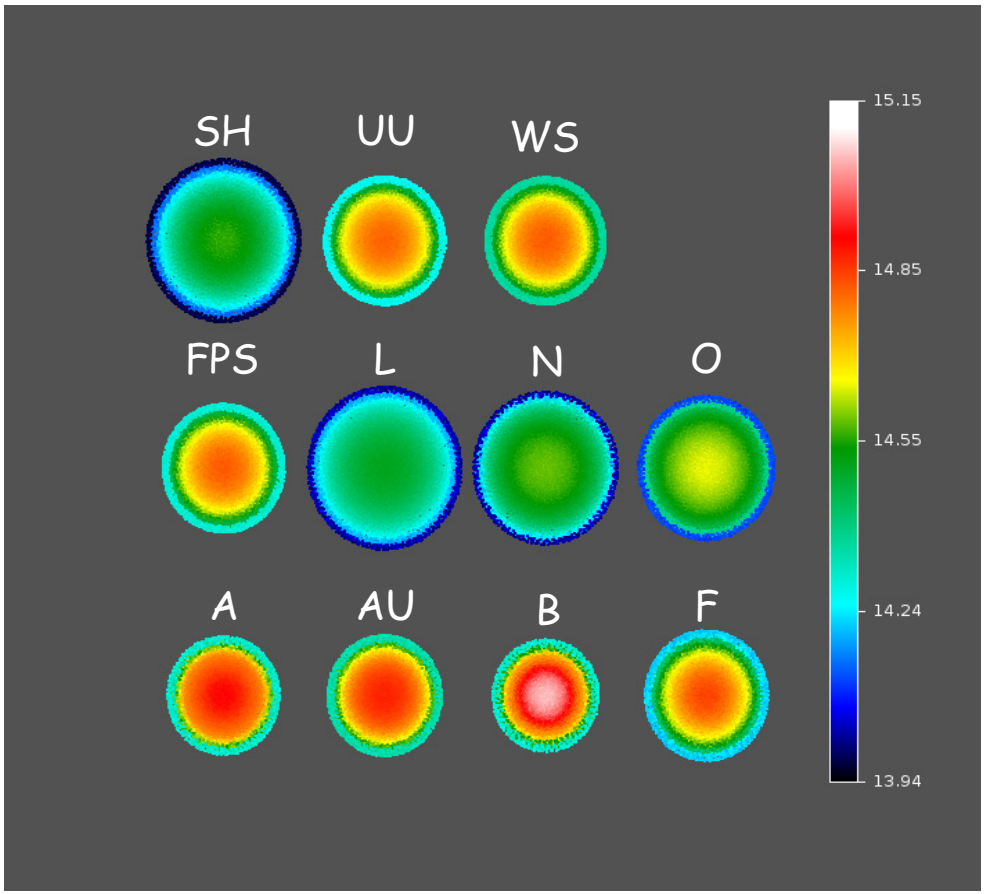

Fig. 2. Equatorial section of the initial models of thirteen neutron stars obtained with different EOS after the hydrodynamical relaxation. Density is represented in colours and every model is labelled with the EOS used for its construction. All stars have $1.4 \mathrm{M}_{\odot}$.

\footnotetext{
${ }^{1}$ A brief description of each EOS and a bibliographic reference can be found on table 2.
} 
mass ratio $\mathrm{q}=1$ for each EOS. In each scenario the initial orbital distance was 1.5 times the Roche lobe overflow distance to ensure that the stars are still within the point-mass inspiral regime [9]. We have to simulate the effect of angular momentum loss due to the emission of gravitational waves in order to induce the coalescence. This is done through an artificial backreaction implemented following the recipe of [1]. This backreaction is simulated as a small but constant frictional deceleration which forces the centers of mass of the neutron stars to follow a point-mass inspiral trajectory. The backreaction term is applied until tidal effects dominate. At that point the hydrodynamics lead naturally to a faster inspiral and coalescence.

\section{Polytropic versus realistic EOS}

Polytropic equations of state are easy to implement and well-behaved, that is why they have been extensively used until now to carry out parametric studies like, for example, the dependence of gravitational wave features (luminosity, secondary peaks) on the stiffness of the EOS. Thus, it was expected that the results could be unambiguously correlated to those obtained with realistic EOS. In order to compare results we need to know if there is a correspondence between polytropic and realistic EOS or, in other words, to find which polytropic exponent $\Gamma$ better describes a particular realistic EOS for the density range relevant for neutron stars. Next we will show three simple methods which attempts to give that correspondence between polytropic and realistic EOS. In all of them we have fixed the total mass of the NS to $1.4 \mathrm{M}_{\odot}$ for simplicity.

It is obvious that in a log-log graphic a polytropic EOS is represented by a straight line of slope $\Gamma$, while realistic EOS have a more complex structure. Thus the most basic approximation is to try a linear fit within a determined range of the realistic EOS which will give the corresponding polytropic exponent $\Gamma_{\text {adj. }}$ The main problem here is that the limits of the adjustment are arbitrary, so we choose $\rho_{\mathrm{c}}$ and $\rho_{95}$ (central density and the density that corresponds to the shell containing the $95 \%$ of the total mass of the NS respectively) as limits for the fit.

A second approach could be to find a mass-weighted "local" polytropic exponent. Hence we have divided the NS in concentric shells and found the local $\Gamma$ through a linear fit of the realistic EOS between the ranges of densities defined by each shell. After that we found the mass that encloses each shell and used it as a weight to average all the local $\Gamma \mathrm{s}$. The resulting polytropic exponent is labelled $\Gamma_{\mathrm{wgt}}$.

The last, and probably more realistic, approach could be to adjust the actual structure of the NS for both types of EOS. However this is not straightforward so we calculated a dense grid of polytropic models with constant mass but varying $\Gamma$ and the central density. With the total mass fixed, we integrated the well-known Lane-Emden equation for each value of $\Gamma$ and found the corresponding radii and polytropic constant. The resulting 3D surface of polytropic models of $1.4 \mathrm{M}_{\odot}$ can be seen in figure 4 . Once the grid was built we used a realistic EOS to obtain the 
radii and central density for that mass. Then we could find which polytropic model of the grid had the same radii and central density, obtaining $\Gamma_{\text {str }}$ for that given realistic EOS.

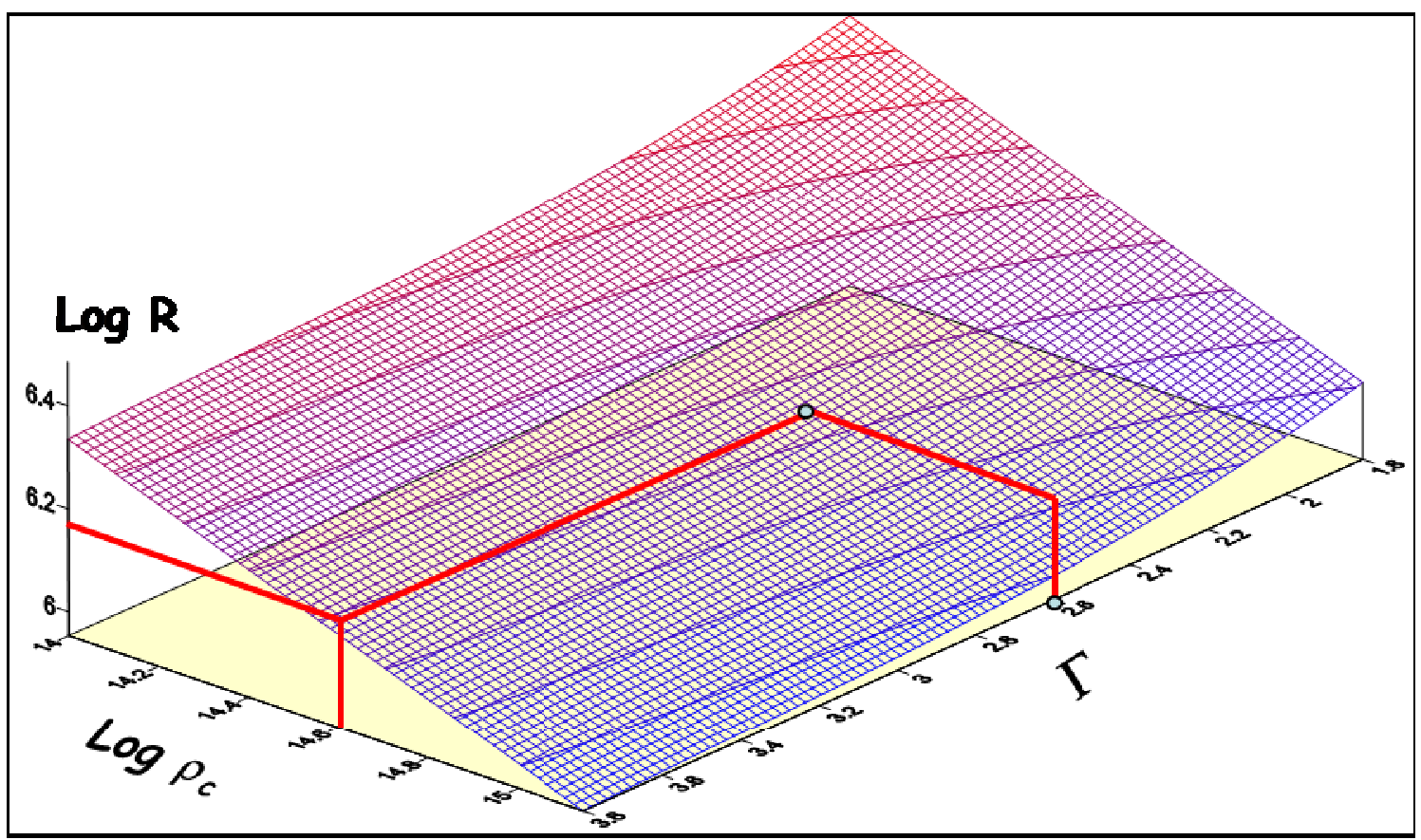

Fig. 4. Mesh of polytropic models for a fixed mass of $1.4 \mathrm{M}_{\odot}$. Red lines denote the procedure to find the corresponding $\Gamma_{\text {str }}$ for given central density and radii from a realistic EOS.

In table 2 the resulting polytropic exponents for the three exposed methods can be found. In the first column the realistic EOS have been ordered from softer to stiffer, using the central density of the model in hydrostatic equilibrium as criteria. As we can see, softer EOS, which corresponds to a more compressible material, are situated in the first rows of the table and stiffer EOS in the last ones. Hence we expect to obtain values of $\Gamma$ ordered from low to high with the three methods explained before. These results can be found in the three last columns of table 2 .

In first place we can see that none of these methods gives the same result for a given realistic EOS. Secondly, at first glance, if we halve horizontally the table it can be seen that the three methods have roughly lower values of $\Gamma$ in the upper half than in the lower, as it would be expected. But if we look closely we can see values that are misplaced. For example, in the last column we can see that the realistic EOS labeled AU has a higher value $(\Gamma=2.50)$ than the one label SH $(\Gamma=2.36)$ which is clearly not the case from the models in hydrostatic equilibrium.

Therefore, questions arise about the credibility of pure polytropic EOS to mimic the hydrodynamical behavior and the gravitational wave emission observed when more complicated EOS are used. Even the notion of soft and stiff EOS, which naturally arises from polytropic EOS, becomes blurred. Nowadays there are good databases concerning realistic EOS so more 
precise studies of gravitational waves radiation can be done with not too much effort in implementation.

In fig. 3 there is a series of snapshots of the plus polarization of the gravitational wave emission of the simulated mergers, obtained with our code, in function of time in dynamical time units, defined by $t_{d}=\left(R^{3} / G M\right)^{1 / 2}$. The letters label the EOS used in the simulation.

\begin{tabular}{|c|c|c|c|c|c|c|c|c|}
\hline \multirow[b]{2}{*}{ EOS } & \multirow[b]{2}{*}{ Ref. } & \multirow[b]{2}{*}{ Many-body theory } & \multicolumn{6}{|c|}{$\mathrm{M}=1.4 \mathrm{M}_{\odot}$} \\
\hline & & & $\rho_{c}\left(10^{14}\right)$ & $R\left(10^{6}\right)$ & $\rho_{95}\left(10^{14}\right)$ & $\Gamma_{\text {adj }}$ & $\Gamma_{\mathrm{wgt}}$ & $\Gamma_{\text {str }}$ \\
\hline B & $\mathrm{AB}$ & Variational Principle & 13.69 & 1.12 & 2.56 & 2.50 & 2.77 & 2.14 \\
\hline A & $\mathrm{AB}$ & Variational Principle & 9.17 & 1.19 & 2.24 & 2.79 & 3.78 & 2.44 \\
\hline $\mathbf{A U}$ & WFF & AV14 + UVII (VM) & 8.12 & 1.22 & 2.22 & 2.93 & 3.32 & 2.50 \\
\hline $\mathbf{F}$ & $\mathrm{AB}$ & Bueckner G-matrix & 7.77 & 1.31 & 1.70 & 2.64 & 2.57 & 2.24 \\
\hline FPS & LRP & UV14 + TNI (VM) & 7.16 & 1.31 & 1.94 & 2.98 & 2.90 & 2.34 \\
\hline WS & WFF & UV14 + TNI (VM) & 7.01 & 1.30 & 2.21 & 3.22 & 3.06 & 2.40 \\
\hline $\mathbf{U U}$ & WFF & UV14 + UVII (VM) & 6.91 & 1.30 & 1.92 & 2.99 & 3.12 & 2.44 \\
\hline $\mathbf{O}$ & $\mathrm{AB}$ & Relativistic density Green func. & 4.65 & 1.44 & 1.41 & 3.37 & 3.99 & 2.60 \\
\hline $\mathbf{N}$ & $\mathrm{AB}$ & Relativistic mean field & 3.94 & 1.53 & 1.24 & 3.44 & 3.64 & 2.59 \\
\hline SH & $\mathrm{S}$ & Relativistic mean field & 3.74 & 1.63 & 0.93 & 2.82 & 2.76 & 2.36 \\
\hline $\mathbf{L}$ & $\mathrm{AB}$ & Mean field; Variational method & 2.49 & 1.82 & 0.83 & 3.01 & 3.47 & 2.47 \\
\hline
\end{tabular}

Table 2. Realistic EOS ordered from softer (top) to stiffer (bottom). The references are AB: [10], WFF: [11], LRP: [12] and S: [13]. The last three columns show the "effective" polytropic exponents obtained with the three methods explained in the text.

As it can be seen each merger has different features. For example, soft EOS like $\mathbf{B}, \mathbf{A}$ or FPS, have higher maximum amplitudes of the waveform in the merger phase than stiffer EOS like $\mathbf{U U}, \mathbf{N}$ or $\mathbf{S H}$. This property leads to a natural ordering of the EOS by its stiffness that is almost coincident with the ordering performed before, based on the models in hydrostatic equilibrium, (columns 4 and 5 of table 2). Also the decay of the gravitational emission after the merger is faster for softer EOS than for stiffer as the formers gain axial symmetry faster due to their higher compressibility, as expected. After a successful gravitational wave detection has been done, all these features could help us to put constraints in the nuclear EOS. Also a helpful tool to interpret future GW detections could be to devise a set of waveform templates calculated through hydrodynamical simulations. That will allow us to learn about microphysics and the behaviour of matter under extreme conditions. 

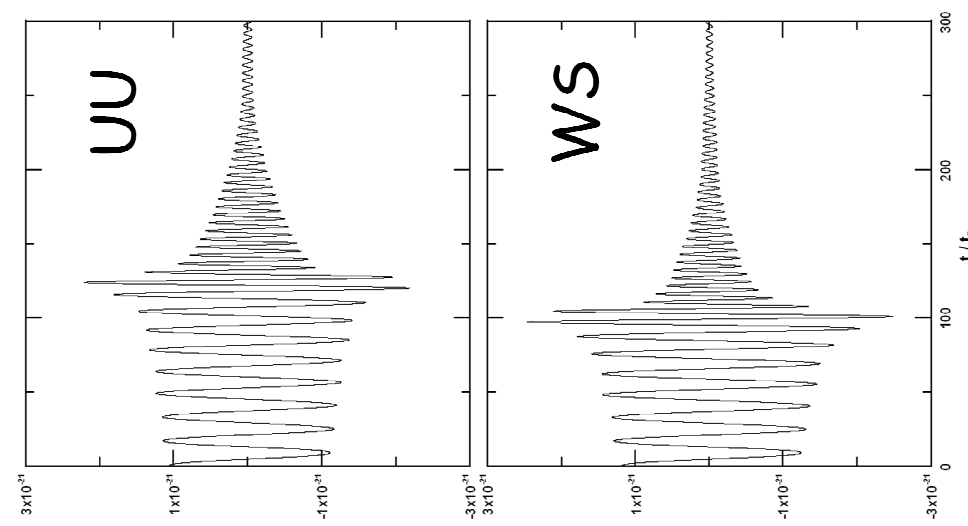

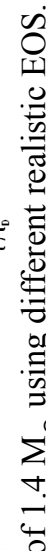
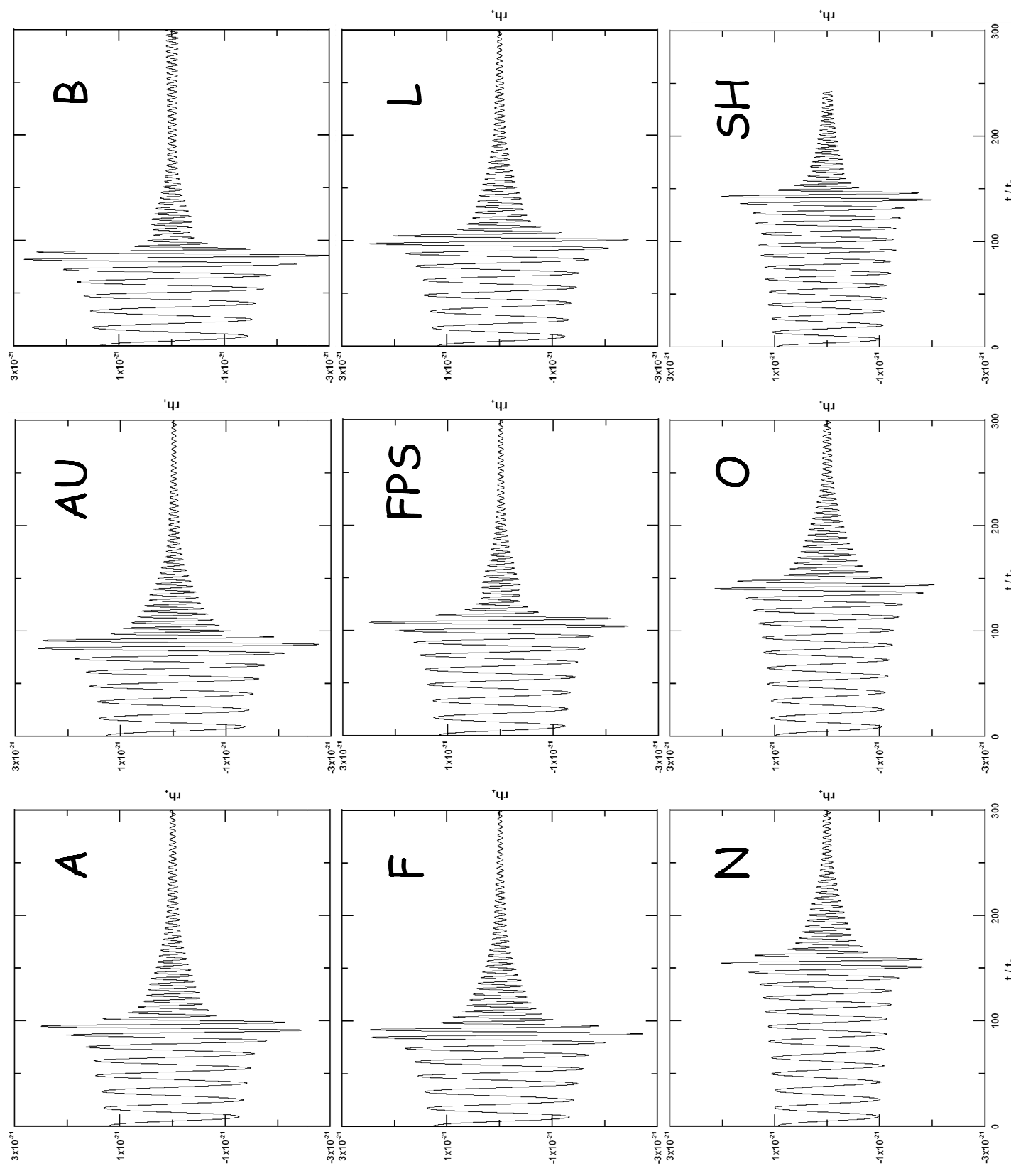

4

$+4$

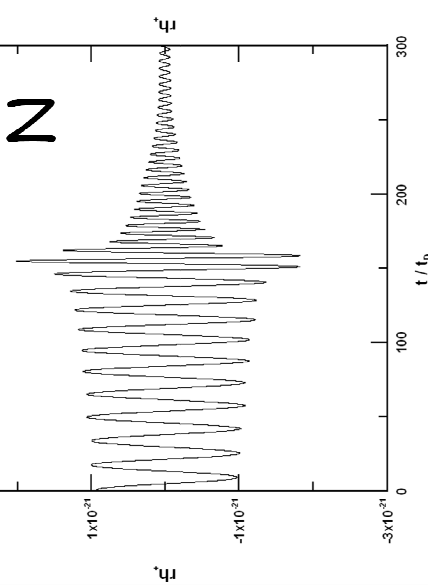




\section{Conclusions}

Gravitational waves can represent an important tool to impose strong constraints in one important cornerstone of modern astrophysics: the nuclear EOS. Taking profit of the existence of scenarios like double neutron star systems, which are powerful emitters of gravitational waves, and that there is a serious promise that current $\mathrm{GW}$ observatories will allow to look for a successful detection, a connection between the EOS of NS matter and its gravitational waves emission though the hydrodynamics involved during a coalescence can be made. To accomplish this, 3D numerical simulations are an important tool to explore the parameter space and to build a complete set of waveforms to use as templates to interpret the observations. In this respect we have demonstrated that the use of polytropic EOS is not recommended if we want consistent physical results in numerical simulations. The dependence of the gravitational waves on the complex structure of the realistic EOS cannot be related to the results obtained with polytropic EOS because there is no correspondence between both types of EOS and we did not find a reliable method to obtain an "effective" polytropic EOS able to mimic the realistic EOS sample. Therefore the utilization of realistic EOS is strongly recommended in any numerical simulation oriented to build waveform templates or study the EOS influence in the gravitational wave emission. Several examples of waveforms obtained through hydrodynamical simulations (in Newtonian approximation) using eleven different realistic EOS were provided in this work.

\section{References}

[1] X. Zhuge, J. M. Centrella \& S. L. W. McMillan, Gravitational radiation from coalescing binary neutron stars, PRD 50 (1994) 6247

[2] K. Belczynski, V. Kalogera \& T. Bulik, A comprehensive study of bynary compact objects as gravitational wave sources: evolutionary channels, rates and physical properties, ApJ 572 (2002) 407 [astro-ph/0111452]

[3] J. J. Monaghan, Smoothed particle hydrodynamics, RPPh 68 (2005) 1703

[4] L. Blanchet, T. Damour \& G. Schäfer, Post-Newtonian hydrodynamics and post-Newtonian gravitational waves generation for numerical relativity, MNRAS 242 (1990) 289

[5] M. Shibata \& K. Uryû, Gravitational waves from the merger of binary neutron stars in a fully general relativistic simulation, PThPh 107 (2002) 265

[6] J. M. Centrella \& S. L. W. McMillan, Gravitational radiation from non axisymmetric collisions of neutron stars, ApJ 416 (1993) 719

[7] C. Misner, K. Thorne \& J. Wheeler, Gravitation (New York: Freeman) 1973

[8] C. Kochanek et al., Gravitational radiation from colliding clusters: Newtonian simulations in three dimensions, ApJ 358 (1990) 81

[9] P. P. Eggleton, Approximations to the radii of Roche lobes, ApJ 268 (1983) 368

[10] W. D. Arnett \& R. L. Bowers, A microscopic interpretation of neutron star structure, ApJS 33 (1977) 415 
[11]R. B. Wiringa, V. Fiks \& A. Fabrocini, Equation of state for dense nucleon matter, PRC 38 (1988) 1010

[12] C.P. Lorenz, D. G. Ravenhall \& C. J. Pethick, Neutron star crusts, PRL 70 (1993) 379

[13]H. Shen et al., Relativistic equation of state of nuclear matter for supernova explosion, PThPh 100 (1998) 1013 